\title{
High-throughput profiling identifies clinically actionable mutations in salivary duct carcinoma
}

\author{
Bo Mi Ku${ }^{1 \dagger}$, Hyun Ae Jung ${ }^{2 \dagger}$, Jong-Mu Sun ${ }^{2}$, Young Hyeh Ko ${ }^{3}$, Han-Sin Jeong ${ }^{4}$, Young-lk Son ${ }^{4}$, Chung-Hwan Baek ${ }^{4}$,
} Keunchil Park ${ }^{2}$ and Myung-Ju Ahn ${ }^{2 *}$

\begin{abstract}
Background: Salivary duct carcinoma (SDC) is a highly aggressive subtype of salivary gland cancers and there is no established standard therapy for this disease. Thus, development of molecular markers for SDC will be important to guide the diagnosis and therapy of this aggressive tumor.

Methods: We performed next-generation sequencing using the Ion Torrent AmpliSeq cancer panel, which explores the mutational status of hotspot regions in 50 cancer-associated genes, and we analyzed copy number variations (CNVs) of 21 genes by NanoString nCounter for 37 patients with SDC. Fluorescent in situ hybridization was also conducted to confirm ERBB2 gene amplification. Clinical records and tumor histopathology of the patients were retrospectively reviewed.

Results: Genetic alterations were detected in 29 of 37 (78.3\%) tumors, including mutations in PIK3CA ( $N=9$, $24.3 \%)$, ERBB2 $(N=4,10.8 \%)$, and EGFR $(N=4,10.8 \%)$. To our knowledge, this is the first time that ERBB2 mutations have been reported in this tumor type. Both PIK3CA and ERBB2 mutation status were associated with poor overall survival, but without statistical significance. ERBB2 amplification was strong and common in SDC and almost all cases also exhibited EGFR and ERBB3 amplifications.

Conclusions: This study reports the largest and most comprehensive analysis of DNA aberrations in SDC. Our results show that PIK3CA and/or ERBB2 alterations in the development of SDC might be a useful diagnostic tool and could serve as a potential therapeutic target.
\end{abstract}

Keywords: Salivary duct carcinoma, Next-generation sequencing, Molecular markers, PIK3CA, ERBB2, EGFR

\section{Background}

Salivary gland cancers (SGC) are rare, accounting for fewer than $5 \%$ of all cancers of the head and neck [1]. $\mathrm{SGC}$ present numerous morphologic, biologic, and clinically diverse entities. Salivary duct carcinomas (SDC) are a rare and aggressive subtype of SGC [2,3]. It bears a pathologic resemblance to ductal carcinoma of the breast and shares hormonal and molecular marker alterations with breast cancer [4]. SDC mainly arises de novo in the parotid gland and most patients are older than 50 years. The recurrence rate is high and metastases occur in lymph

\footnotetext{
* Correspondence: silk.ahn@samsung.com

${ }^{\dagger}$ Equal contributors

${ }^{2}$ Division of Hematology-Oncology, Department of Medicine, Samsung Medical Center, Sungkyunkwan University School of Medicine, 50 Irwon-dong, Gangnam-gu, Seoul 135-710, Korea

Full list of author information is available at the end of the article
}

nodes, lung, liver, and bone [5]. Despite surgical resection and postoperative radiotherapy, SDC has an extremely poor prognosis $[2,4]$ and most patients with SDC die from progressive disease within 3 years $[2,6]$.

To date, several chemotherapeutic agents have been tested but none have shown significant efficacy [7]. Although there are limited prior molecular studies of SDC, many of these studies have suggested that overexpression of epidermal growth factor receptor 2 (ERBB2, also known as HER2) is a common event in this tumor type. Expression of ERBB2 has been linked to early local disease recurrence, distant metastasis, and poor survival $[2,8]$. In spite of the high incidence of ERBB2 gene amplification, as detected by fluorescence in situ hybridization (FISH), there has been no meaningful or reproducible response to ERBB2-targeted therapy [4,9]. Furthermore, given the rarity of the disease, comprehensive analyses of 
biomarkers are limited. Thus, identifying the molecular genetic alterations in SDC should provide new opportunities for the development of therapeutics.

Significant improvements in sequencing technology and computational methods have led to the development of next-generation sequencing (NGS) platforms. Several NGS platforms are available for sequencing either targeted genomic regions or whole genomes/exomes to analyze a variety of disease-associated changes such as point mutations, insertions, deletions, and copy number variations (CNVs) [10]. These broad NGS applications have facilitated the discovery of novel genetic aberrations in disease and NGS may be a useful tool for mutation screening for diagnosis and discovery [11].

In the present study, we used an AmpliSeq cancer panel and NanoString nCounter assay to determine cancerassociated mutations and CNVs in 37 SDC patients. CNVs of ERBB2 that were detected using NanoString nCounter assay were confirmed using FISH.

\section{Methods}

Tumor specimen and DNA extraction

All procedures involving tumor specimen were reviewed and approved by the Institutional Review Board (IRB) of Samsung Medical Center (SMC). Patient records/information was anonymized and de-identified prior to analysis. We retrospectively identified 48 patients who were diagnosed with SDC at Samsung Medical Center from January 1997 to April 2010. A total of 37 cases of SDC had adequate tissue available for DNA isolation. Genomic DNA was extracted from formalin-fixed paraffinembedded tissue (FFPE) using the QIAamp DNA FFPE Tissue Kit (Qiagen). Purified DNA was quantitated using NanoDrop (Invitrogen Life Technologies) and Qubit (Invitrogen Life Technologies).

\section{Next-generation sequencing}

Ion Torrent Ion AmpliSeq Cancer Hotspot Panel v2 (Life Technologies) was used to sequence hotspot regions in 50 frequently mutated tumor suppressor genes and oncogenes. For multiplex PCR amplification, $10 \mathrm{ng}$ of DNA (quantified by Qubit ${ }^{\mathrm{m}}$ Fluorometer) was used and the custom Ion AmpliSeq panel was processed with Ion AmpliSeq Library kit 2.0 according to the manufacturer's instructions. The resulting amplicons were treated with $\mathrm{FuPa}$ Reagent to partially digest the primers and phosphorylate the amplicons. The amplicons were then ligated to the Ion Xpress ${ }^{\text {Tx }}$ Barcode Adapters (1-96 Kit) and template preparation was performed with the Ion OneTouch $^{\text {Tx }}$ System using Ion OneTouch 200 Template Kit v2 DL. Sequencing was performed on Ion 316 chips using the Ion PGM $^{\text {sx }} 200$ Sequencing Kit according to the manufacturer's instructions. The raw signal data were analyzed using Torrent Suite v.4.0.2 (Life Technologies).
The pipeline includes signaling processing, base calling, quality score assignment, adapter trimming, read alignment (Torrent Mapping Alignment Pregram; TMAP) to human genome (HG) 19 reference, mapping quality control, and coverage analysis. Variant calling was performed using the Torrent variant Caller 4.0 software. COSMIC

\section{Table 1 Baseline characteristics}

\begin{tabular}{|c|c|}
\hline Total $(\mathrm{N}=48)$ & Patient (N) \\
\hline Age (Median/Range) & $59.3(39.3-81.6)$ years \\
\hline Sex (Male : Female) & $36: 12$ \\
\hline \multicolumn{2}{|l|}{ Location } \\
\hline Parotid gland & 38 \\
\hline Submandibular gland & 10 \\
\hline \multicolumn{2}{|l|}{ Stage } \\
\hline I & 4 \\
\hline$\|$ & 17 \\
\hline III & 6 \\
\hline IV & 21 \\
\hline \multicolumn{2}{|l|}{ Treatment } \\
\hline Operation & 3 \\
\hline Operation + RT & 25 \\
\hline Radiation dose (Median/Range) & 5400 cGy (4000-6300 cGy) \\
\hline Operation + CCRT & 14 \\
\hline Radiation dose (Median/Range) & 5940 cGy (5400-6000 cGy) \\
\hline \multicolumn{2}{|l|}{ Chemotherapy regimen } \\
\hline Cisplatin & 1 \\
\hline $\mathrm{DP}$ & 5 \\
\hline $\mathrm{FP}$ & 2 \\
\hline CAP & 4 \\
\hline Unknown & 2 \\
\hline CCRT & 1 (Genexol/cisplatin) \\
\hline Palliative chemotherapy & $2(C A P, X P)$ \\
\hline Palliative radiotherapy & 4 \\
\hline \multicolumn{2}{|l|}{ Treatment response } \\
\hline No evidence disease & 23 \\
\hline Stable disease & 1 \\
\hline Recur or Progression & $19 / 5$ \\
\hline Recurrence sites & Total $(\mathrm{N}=19)$ \\
\hline Locoregional recurrence & 8 \\
\hline Lung & 6 \\
\hline Bone & 4 \\
\hline Liver & 2 \\
\hline RFS (Median) & 29.1 months \\
\hline OS (Median) & 76.2 months \\
\hline
\end{tabular}

RT: Radiotherapy; CCRT: Concurrent chemo-radiotherapy; DP: Docetaxel/Cisplatin; FP: 5FU/Cisplatin; CAP: Cyclophosphamide/Doxorubicin/Cisplatin; RFS: Recurrence-free survival; OS: Overall survival. 
DB, dnsnp137, and annovar were used as annotation program.

\section{Copy number alteration analysis}

For detection of CNVs in salivary ductal carcinoma, a panel of customized gene probes was designed using NanoString nCounter technology and subsequently analyzed on the NanoString nCounter platform. NanoString probes were composed of the following 21 genes (in alphabetical order): AURKA, CCND1, CCNE1, CDK4, CDK6, CDKN1A, CDKN2A, EGFR, ERBB2, ERBB3, FGFR1, FGFR2, IGF1R, KLF5, KRAS, MDM2, MET, MITF, $M Y C$, PIK3CA, TNIK. For the nCounter assay, $600 \mathrm{ng}$ of genomic DNA was hybridized with the custom designed code set for 18 hours at $65^{\circ} \mathrm{C}$ and processed according to the manufacturer's instruction. The data were normalized to the invariant control probes and to positive and negative controls in each hybridization reaction.
Fluorescence in situ hybridization

To evaluate copy number changes in the ERBB2/HER2 gene, a 4- $\mu \mathrm{m}$ section of FFPE tumor tissue was analyzed by dual-color FISH using the PathVysion HER2 DNA Prove Kit (Abbott Molecular), which includes HER2 Spectrum Orange and centromere 17 (CEP17) Spectrum Green.

\section{Statistical analysis}

Patient characteristics were compared using chi-square and Fisher's exact tests (categorical variables). Survival time was estimated using the Kaplan-Meier method and compared for statistical differences by log-rank analysis. Multivariate analysis was performed using stepwise cox proportional hazards regression modeling to assess the independent prognostic role of each clinicopathologic variable. Statistical analyses were performed using SPSS19.0 (SPSS Inc.) and statistical significance was considered to be $p<0.05$.

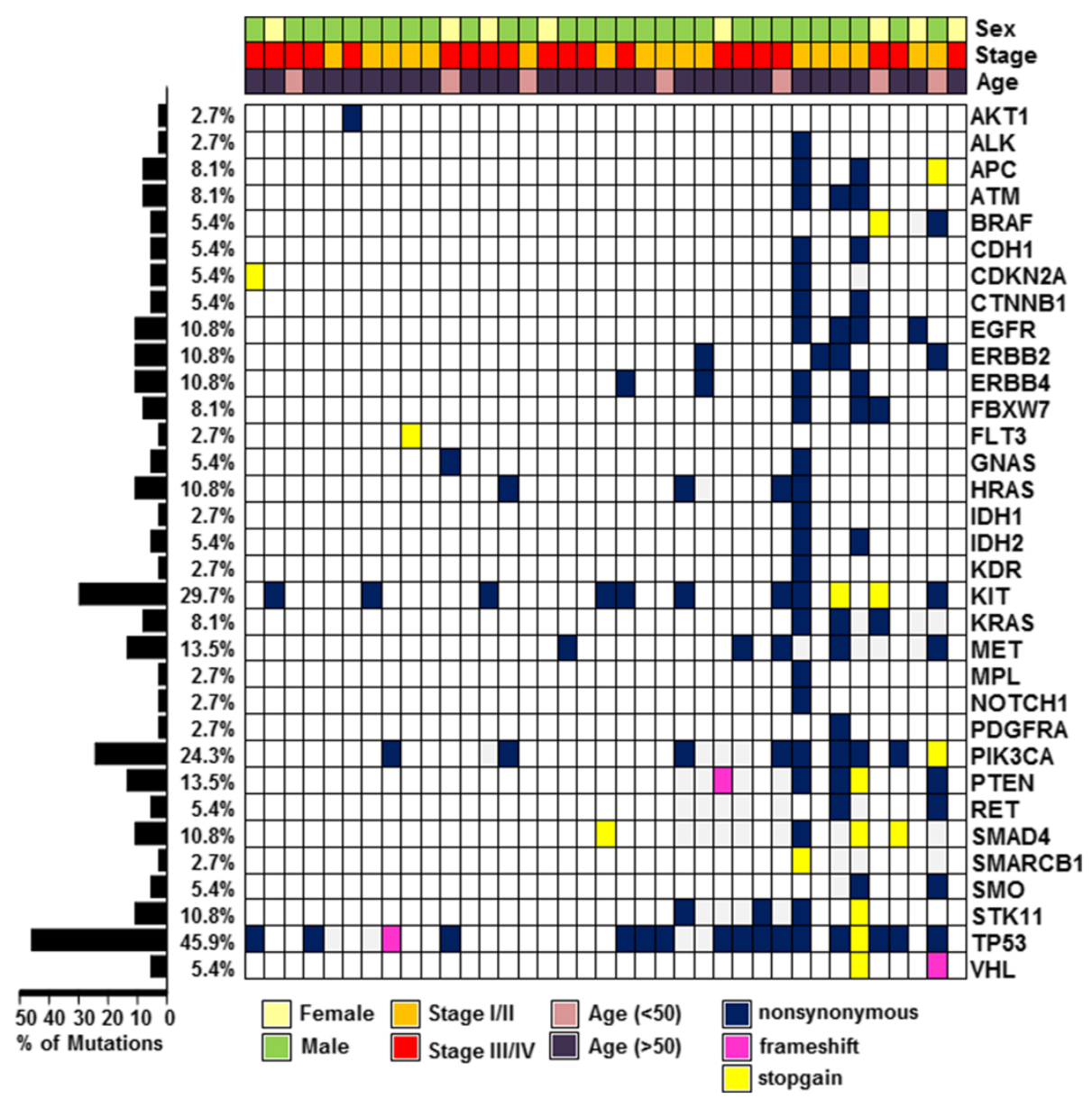

Figure 1 Heatmap of the mutations found in $\mathbf{3 7}$ SDC samples. In the upper panel, the first row indicates sex, the second row cancer stage, and the third row age. (Left) the histogram shows the percentage of mutations in each gene. (Right) The horizontal axis presents the complete dataset of patients and the vertical axis illustrates mutated genes. 


\section{Results}

\section{Clinical characteristics of the cohort}

Characteristics of all 48 patients are listed in Table 1. The median age was 59.3 years (range 39.3-81.6 years) and male patients were predominant $(75 \% ; 36 / 48)$. The primary tumor site was the parotid gland in 38 (80\%) cases and the submandibular gland in 10 cases (20\%). Regarding tumor stage, the majority (44\%) of patients presented with stage IV disease. Surgery was performed for 41 patients (85.4\%) and postoperative radiotherapy and/or chemotherapy were performed for 24 (50\%) and 14 (29\%) cases, respectively. Among patients who did not undergo surgery, 1 case was treated with concurrent chemo-radiotherapy, 2 cases with palliative chemotherapy, and 4 cases with palliative radiotherapy. Among 42 patients who underwent surgery, 19 cases (45.2\%) had recurrence of disease at either locoregional or distant sites and 23 cases (54.8\%) showed no evidence of disease. With a median follow-up of 44.7 months (range: 3.7-214.0 months), the median recurrence-free survival (RFS) time was 17.3 months (95\% CI, 14.2-20.4) and median overall survival (OS) time was 76.2 months $(95 \%$ CI, 15.7-136.7). Among the 48 patients, only 37 cases of SDC had adequate tissue available for DNA isolation.

\section{Molecular profiling of SDC}

To evaluate genetic abnormalities occurring in SDC, we applied NGS technology using the Ion Torrent Ion AmpliSeq Cancer Hotspot Panel. Among the detected mutations, only mutations annotated in the Catalogue of Somatic Mutations in Cancer (COSMIC) database were considered. Twenty-nine of 37 (78.3\%) patients possessed more than one mutation in this analysis (Figure 1). Genes with identified mutations included $A P C$ (8 mutations), ATM (3), EGFR (7), ERBB2 (4), ERBB4 (5), FBXW7 (3), HRAS (2), KIT (7), KRAS (4), MET (1), PIK3CA (15), PTEN (10), SMAD4 (10), STK11 (4), and TP53 (33) (Table 2). With the exception of TP53 and KIT mutations, the most frequently identified mutations were in PIK3CA (15 mutations in 9 patients). Three hotspot mutations (E542K, E545K, and H1047R) of PIK3CA were observed in $13.5 \%$ of patients $(5 / 37)$. Although seven EGFR mutations were detected, there were no cases with activating EGFR mutations (exon 19 deletion or L858R), which are well-known gene abnormalities in non-small cell lung cancer. Interestingly, in addition to ERBB2 amplification, which is known to be a common event in SDC [4], our results also revealed $E R B B 2$ mutations in four patients at four different loci: I767M, D769Y, G776V, and V842I. The KRAS mutations identified in this study were not in codons G12, G13, or Q61, which impair the intrinsic GTPase activity of RAS and sustain the activation of RAS signaling [12].

The CNVs were also tested and validated by using NanoString nCounter technology. The results showed that 14 of the 21 genes had CNV, including both copy number amplifications and losses. These genes included CCND1 (83.7\%), CDK4 (91.8\%), CDKN1A (100\%), CDKN2A (35.1\%), EGFR

Table 2 Mutations found in 37 SDCs by AmpliSeq

\begin{tabular}{|c|c|c|c|}
\hline Gene & Accession & $\begin{array}{l}\text { Number of } \\
\text { mutations }\end{array}$ & Mutations \\
\hline$\overline{A P C}$ & NM_000038 & 8 & G1106R, A1347T, P1361L, E1374K, Q1378X, S1434N, G1466E, H1490Y \\
\hline ATM & NM_000051 & 3 & E848K, D1693N, A1742V \\
\hline EGFR & NM_005228 & 7 & V765M, A859T, A864V, E865K, E866K, A871T, G874S \\
\hline ERBB2 & NM_004448 & 4 & I767M, D769Y, G776V, V842I \\
\hline ERBB4 & NM_005235 & 5 & A287T, S341L, D609N, R612Q, P619L \\
\hline FBXW7 & NM_033632 & 3 & G391D, D440N, R441W \\
\hline HRAS & NM_176795 & 2 & G13S, Q61R \\
\hline $\mathrm{KIT}$ & NM_000222 & 7 & D496N, M541L, W557X, E561K, W582X, A636V, A637F \\
\hline KRAS & NM_004985 & 4 & G15S, T35I, E49K, E63K \\
\hline MET & NM_000245 & 1 & N375S \\
\hline PIK3CA & NM_006218 & 15 & $\begin{array}{l}\text { E65K, R88X, R108H, E110K, S326F, V344M, D350N, E542K, E545K, D1018N, } \\
\text { A1020T, E1037K, M1043I, H1047R, G1049D }\end{array}$ \\
\hline PTEN & NM_000314 & 10 & W111X, D115N, G132S, M134I, R233X, R234Q, Q245X, E256K, T319fs, P339L \\
\hline SMAD4 & NM_005359 & 10 & Q116X, R135X, Q256X, P356S, P356L, R496H, R497H, C499Y, W509X, R531W \\
\hline STK11 & NM_000455 & 4 & Q170X, G171S, P281L, F354L \\
\hline TP53 & NM_001126112 & 33 & $\begin{array}{l}\text { A78V, P80L, A86V, S95F, V97A, P98L, R110C, S127fs, P128fs, K132R, C135W, } \\
\text { V157G, R181C, Q192X, H193Y, R213Q, S215R, P219S, P223S, G226D, S241F, } \\
\text { V272G, R273C, A276D, D281N, R282W, E285K, E286G, E287K, R290H, } \\
\text { G293R, G302E, E339K }\end{array}$ \\
\hline
\end{tabular}


(81\%), ERBB2 (100\%), ERBB3 (81\%), IGF1R (37.8\%), KLF5 (86.4\%), KRAS (100\%), MET (64.8\%), MITF (43.2\%), MYC (72.9\%), and PIK3CA (45.9\%) (Table 3). Of note, ERBB2 exhibited the highest prevalence of strong gene amplification in SDC (Figure 2A). Using FISH, we confirmed ERBB2 gene amplification in 29 cases of SDC. Representative images of SDC cases exhibiting ERBB2 gene amplification are illustrated in Figure 2B. As all patients in this cohort had $E R B B 2$ gene amplification (copy number $>2$ ), we classified these patients into moderate (copy number <10) and strong (copy number $>10$ ) amplification of ERBB2. When survival was analyzed according to amplification status of $E R B B 2$, there was no difference in disease-free survival and overall survival between the two groups (Figure 2C).

\section{Impact of molecular marker status on clinical outcomes} Given the high incidence of PIK3CA or ERBB2 gene abnormalities in this cohort, we investigated the association between molecular status and survival. Kaplan-Meier survival curves were plotted according to PIK3CA or $E R B B 2$ mutation status (Figure 3). In the case of PIK3CA (Figure $3 \mathrm{~A}$ ) or ERBB2 (Figure $3 \mathrm{~B}$ ) mutation, both disease-

Table 3 The frequency CNV in 37 SDCs by NanoString nCounter analysis

\begin{tabular}{|c|c|c|c|c|}
\hline Gene & Case no. & $\begin{array}{l}\text { Copy number } \\
\text { amplification } \\
\text { no. (\%) }\end{array}$ & $\begin{array}{l}\text { Copy number } \\
\text { loss no. (\%) }\end{array}$ & $\begin{array}{l}\text { Total CNV } \\
\text { frequency } \\
\text { no. (\%) }\end{array}$ \\
\hline AURKA & 37 & $0(0)$ & $4(10.8)$ & $4(10.8)$ \\
\hline CCND1 & 37 & 31 (83.7) & $0(0)$ & 31 (83.7) \\
\hline CCNE1 & 37 & $2(5.4)$ & $5(13.5)$ & 7 (18.9) \\
\hline CDK4 & 37 & 34 (91.8) & $0(0)$ & 34 (91.8) \\
\hline CDK6 & 37 & $6(16.2)$ & $4(10.8)$ & $10(27)$ \\
\hline CDKN1A & 37 & $37(100)$ & $0(0)$ & 37 (100) \\
\hline CDKN2A & 37 & $10(27)$ & $3(8.1)$ & $13(35.1)$ \\
\hline EGFR & 37 & $30(81)$ & $0(0)$ & $30(81)$ \\
\hline ERBB2 & 37 & 37 (100) & $0(0))$ & 37 (100) \\
\hline ERBB3 & 37 & $30(81)$ & $0(0)$ & $30(81)$ \\
\hline FGFR1 & 37 & 8 (21.6) & $1(2.7)$ & $9(24.3)$ \\
\hline FGFR2 & 37 & $3(8.1)$ & $2(5.4)$ & $5(13.5)$ \\
\hline IGF1R & 37 & $0(0)$ & $14(37.8)$ & $14(37.8)$ \\
\hline KLF5 & 37 & 32 (86.4) & $0(0)$ & 32 (86.4) \\
\hline KRAS & 37 & 37 (100) & $0(0)$ & 37 (100) \\
\hline MDM2 & 37 & $5(13.5)$ & $2(5.4)$ & 7 (18.9) \\
\hline MET & 37 & $24(64.8)$ & $0(0)$ & $24(64.8)$ \\
\hline MITF & 37 & $0(0)$ & $16(43.2)$ & $16(43.2)$ \\
\hline MYC & 37 & 27 (72.9) & $0(0)$ & 27 (72.9) \\
\hline PIK3CA & 37 & $3(8.1)$ & $14(37.8)$ & $17(45.9)$ \\
\hline TNIK & 37 & $5(13.5)$ & $4(10.8)$ & $9(24.3)$ \\
\hline
\end{tabular}

Copy number amplification: copy number $>2$.

Copy number loss: copy number $<2$. free survival (DFS) and overall survival (OS) curves shifted down. Although there was a tendency toward lower survival in patients with gene mutation (median OS: 76.2 months for wild-type vs. 20.8 months for PIK3CA mutation; 70.9 months for wild-type vs. 20.8 months for $E R B B 2$ mutation), the association was not statistically significant.

\section{Discussion}

Salivary duct carcinoma is an aggressive subtype of salivary gland cancers with histologic similarities to ductal breast carcinoma. Although patients can receive effective therapy including surgical resection followed by radiation therapy or chemotherapy, the high recurrence rate results in poor prognosis [4,7]. The most active single agents for SDC include cisplatin, cyclophosphamide, doxorubicin, and 5-FU. Response rates for combination chemotherapy are reported to be $15-50 \%$ [7]. Given the lack of promising therapeutics in SDC, in this study we aimed to investigate molecular features that can predict responses to therapy and characterize molecular markers for SDC by evaluating genetic status.

Consistent with previous studies, our patient population consisted mostly of males (75\%) older than 50 years, who presented with advanced disease (stage IV: 44\%). In this cohort, we identified a number of mutations and CNVs in several genes. Among these, oncogenic PIK3CA showed a high incidence of mutations in SDC patients (24.3\%). The PI3K axis plays a critical role in tumorigenesis and the availability of small molecular inhibitors of this pathway renders it an attractive therapeutic target [13]. Recently, somatic mutations in PIK3CA have been identified in a variety of human tumors. Most of the reported mutations of PIK3CA are clustered in exons 9 and 20 of the gene $[14,15]$, where three hotspot mutations (E542K, E545K, and H1047R) reside. Two of these hotspot mutations (E542K and E545K) are located in the helical domain (exon 9) whereas H1047R is located in the kinase domain (exon 20) of the PIK3CA protein. All three hotspot mutations have been proven to be oncogenic and are associated with poor clinical outcomes [16-18]. In our study, five patients had hotspot mutations in PI3KCA (one each with E542K and E545K, and three with H1047R). These results are consistent with recent studies that reported PIK3CA mutations in SDC [19-21]. In addition to gain-of-function mutations, PIK3CA is activated through gene amplifications in several cancers $[18,22,23]$. However, the nCounter CNV assay revealed no significant amplification of PIK3CA in SDC patients in this study (Figure 2). In addition to PIK3CA alterations, the phosphatase and tensin homolog (PTEN) also activates the PI3K pathway, and PTEN deletion was recently reported in SDC by FISH analysis $[21,24]$. We found 10 PTEN mutations in 5 out of 37 cases of SDC. Because of the limited 
A

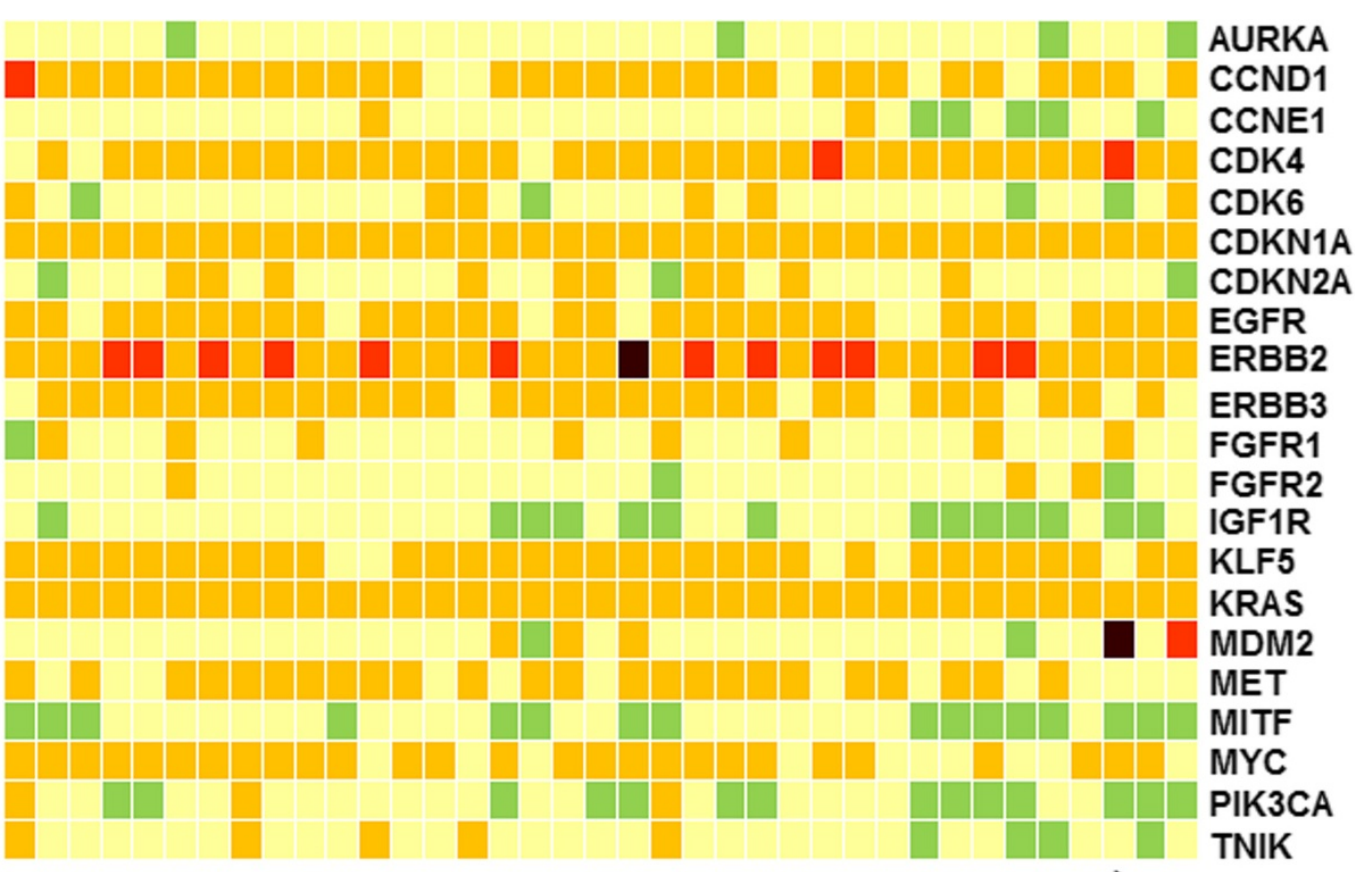

B
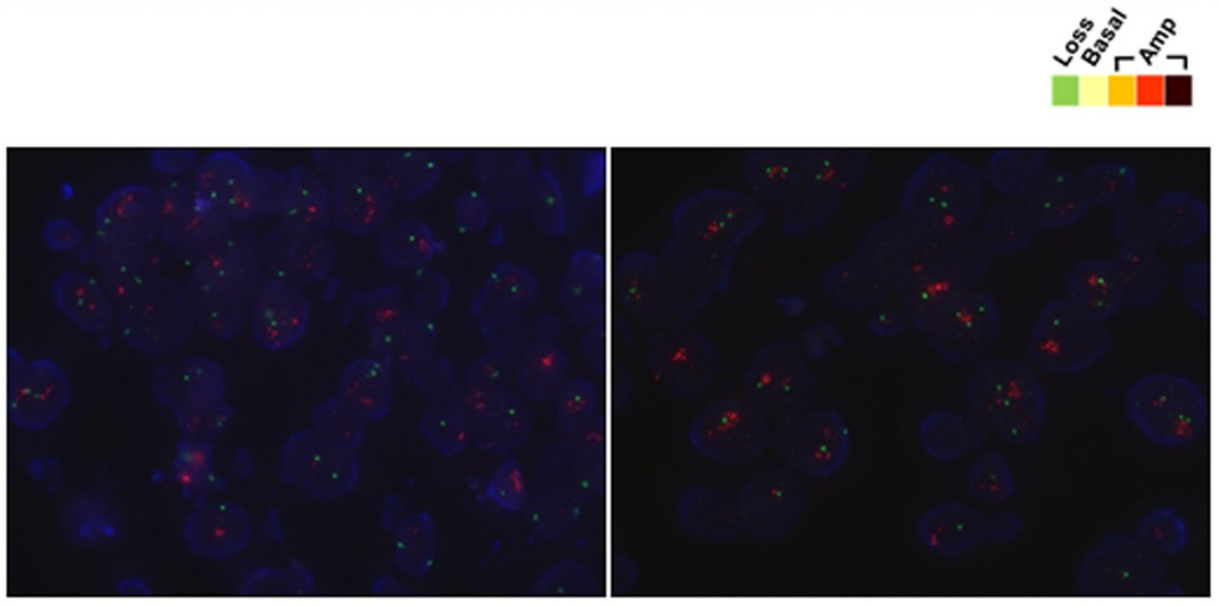

C

Disease-free survival

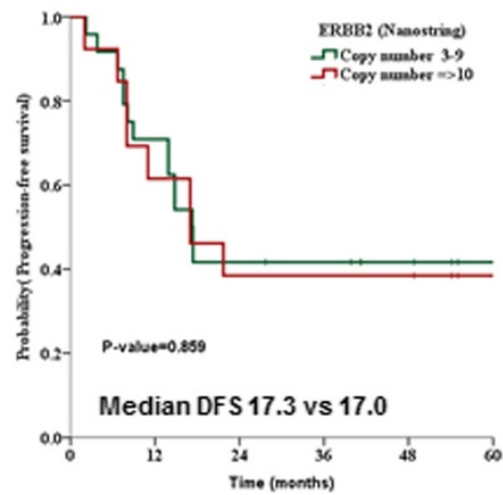

Overall survival

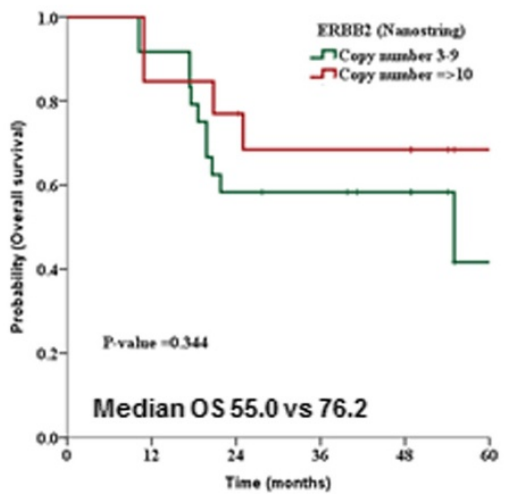

Figure $\mathbf{2}$ (See legend on next page.) 
(See figure on previous page.)

Figure 2 Copy number variations in SDC. A. Copy number variations of 37 SDC samples by the NanoSting nCounter Cancer Copy Number Variation Panel. The horizontal axis represents the complete dataset of patients and the vertical axis indicates examined genes. B. ERBB2 gene amplifications were confirmed by FISH analysis, showing clusters of red signals (ERBB2 probe) and 2 green probes (chromosome 17) per nucleus (DAPI staining). C. Disease-free survival (left) and overall survival (right) depicted according to the ERBB2 gene amplification. Patients grouped by strong (copy number $\geq 10$ ) and moderate (copy number $<10$ ) amplification of ERBB2.

amount of available tissue we could not determine whether these alterations of PIK3CA or PTEN are associated with aberrant protein expression and subsequent activation of the PI3K pathway. However, a previous study reported that PIK3CA amplification appears to be exceedingly rare in SDC, and that PIK3CA mutation may be seen simultaneously with PTEN loss [21]. Further study is required to assess whether PIK3CA mutation could be the predictor of poor prognosis in SDC.

In addition to the histologic resemblance, SDC is similar to breast ductal carcinoma regarding overexpression of ERBB2 and EGFR in many cases [25-30]. ERBB2 gene amplification and protein overexpression occurs in 20-30\% of breast cancers and is reported to be a significant predictor of poor prognosis [31]. Similarly, the majority of cases of SDC appear to strongly overexpress ERBB2. Consistent with previous studies, the majority of our cohort exhibited strong (copy number $\geq 10$ ) amplification of $E R B B 2$. The high concordance between immunohistochemistry and FISH analyses suggests that gene amplification is the most important mechanism for ERBB2 overexpression in this tumor $[25,28,32,33]$. These findings all point to a potential role for trastuzumab, an anti-ERBB2 antibody, in the treatment of SDC. Several studies have reported clinical results of treatment with trastuzumab in combination with chemotherapy $[9,30,34]$. In these studies, selected SDC cases

\section{A Disease-free survival}

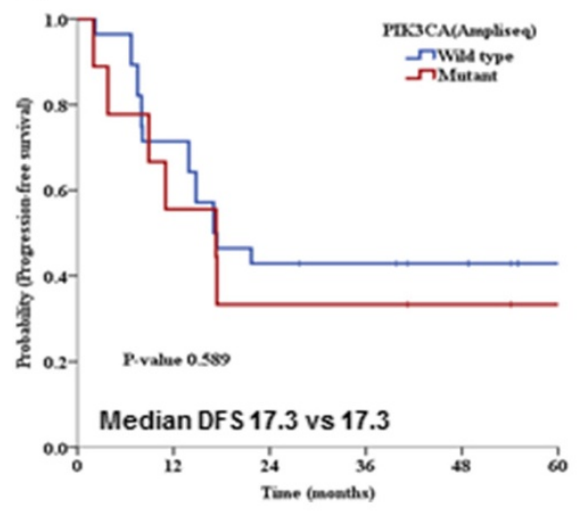

\section{B Disease-free survival}

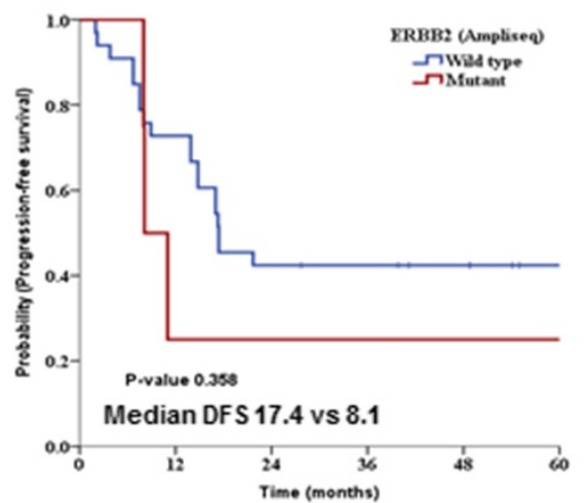

Overall survival

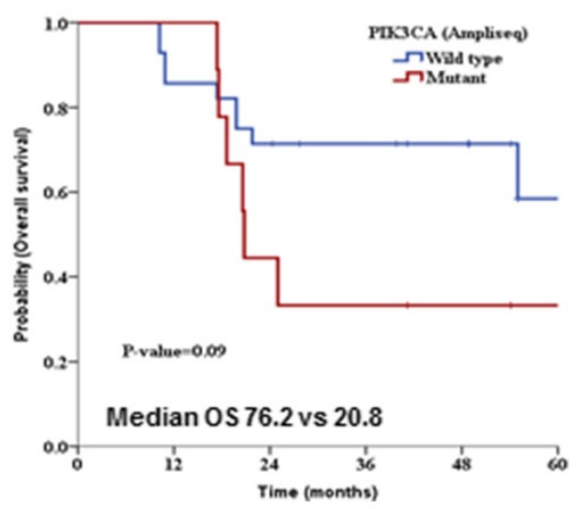

\section{Overall survival}

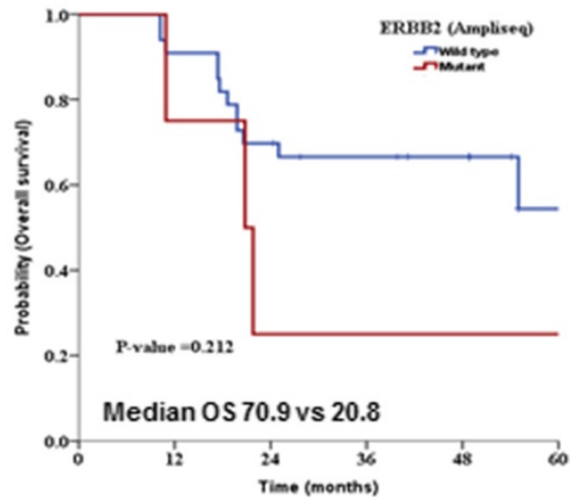

Figure 3 Kaplan-Meier curves for disease-free survival (DFS) and overall survival (OS) analysis. Observed survival of SDC patients is depicted according to the presence or absence of genetic abnormalities: A. mutations in PIK3CA and B. mutations in ERBB2. Statistical analysis revealed no significant differences but indicated a trend toward better survival for patients without genetic mutation. 
with $E R B B 2$ amplification successfully treated with trastuzumab. However, until now ERBB2 mutations had not been identified in patients with SDC. It is therefore noteworthy that we found $E R B B 2$ mutations in 4 of 37 patients. All these mutations were in exons 17-19 (Table 2). Although the functional relevance of these mutations has not been identified, patients with $E R B B 2$ mutation had a shorter median OS time than those without (20.8 vs. 70.9 months). This finding suggests that in a subset of SDC patients, the presence of $E R B B 2$ mutation represents a more attractive potential therapeutic target than $E R B B 2$ amplification.

It has been reported that EGFR overexpression is a poor prognostic factor in head and neck squamous cell carcinoma $[35,36]$. Moreover, cetuximab, an anti-EGFR monoclonal antibody, and/or gefitinib, an ATP-competitive EGFR tyrosine kinase inhibitor (TKI), have been studied in various types of salivary gland carcinoma [4,37-39], but not in SDC. EGFR activation and downstream signaling leads to increased cell proliferation and survival. Activating EGFR mutations are well described in non-small cell lung cancer and have been reported in various cancers of the salivary glands $[25,39,40]$. EGFR mutations are usually localized within two hotspots in the kinase domains, specifically a deletion in exon 19 and an L858R point mutation in exon 21. In addition, rare mutations have been discovered elsewhere in the kinase domain, including insertions in exon 20 [29]. In this study, we observed 7 EGFR mutations in 4 of 37 SDC patients. One of these mutations (V765M) is in exon 20 and the others (A859T, A864V, E865K, E866K, A871T, and G874S) are in exon 21. Although those are not well-known hotspot mutations, their close proximity to L858R suggests that they may play a role in SDC progression.

\section{Conclusions}

In conclusion, we identified mutations in several genes including PIK3CA, ERBB2, and EGFR and significant gene amplification of ERBB2 in SDC. Given the small number of cases in this study, the significance of specific mutation types, mechanism of gene amplification, and their relationship to other abnormalities has to be elucidated in larger studies. In Kaplan-Meier analysis using PIK3CA and ERBB2 genes, the presence of mutations showed a higher correlation with overall survival than copy number variation. Although the importance of these mutations in SDC is still unchallenged, our results suggest that further studies of these mutations may identify a new therapeutic candidate and aid development of an effective treatment strategy in SDC.

\section{Consent}

Written informed consent was obtained from the patient for the publication of this report and any accompanying images.

\section{Abbreviations}

SDC: Salivary duct carcinoma; CNV: Copy number variation; SGC: Salivary gland cancer; ERBB2: Epidermal growth factor receptor 2; FISH: Fluorescence in situ hybridization; NGS: Next-generation sequencing; FFPE: Formalin-fixed paraffin-embedded tissue; COSMIC: Catalogue of Somatic Mutations in Cancer; DFS: Disease-free survival; PTEN: Phosphatase and tensin homolog; TKI: Tyrosine kinase inhibitor; RT: Radiotherapy; CCRT: Concurrent chemo-radiotherapy; DP: Docetaxel/Cisplatin; FP: 5FU/Cisplatin; CAP: Cyclophosphamide/Doxorubicin/Cisplatin; RFS: Recurrence-free survival; OS: Overall survival.

\section{Competing interests}

The authors declared that they have no competing interests.

\section{Authors' contributions}

All authors have contributed to the study design, the acquisition of data (clinical and experimental), or the analysis and interpretation of data. Specifically, JS, YHK, HJ, YS, CB, KP, and MA primarily performed sample and clinical data collection. BMK, HAJ and MA contributed to the study design and conception, performed data analysis and drafted the manuscript. All authors read and approved the final version of the manuscript.

\section{Acknowledgements}

This work was supported by Samsung Medical Center grant [CRS 1104821].

\section{Author details}

${ }^{1}$ Samsung Biomedical Research Institute, Seoul, Korea. ${ }^{2}$ Division of Hematology-Oncology, Department of Medicine, Samsung Medical Center, Sungkyunkwan University School of Medicine, 50 Irwon-dong, Gangnam-gu, Seoul 135-710, Korea. ${ }^{3}$ Department of Pathology, Samsung Medical Center, Sungkyunkwan University School of Medicine, Seoul, Korea. ${ }^{4}$ Department of Otorhinolaryngology-Head and Neck Surgery, Samsung Medical Center, Sungkyunkwan University School of Medicine, Seoul, Korea.

Received: 31 July 2014 Accepted: 14 October 2014

Published online: 25 October 2014

References

1. Laurie SA, Licitra L: Systemic therapy in the palliative management of advanced salivary gland cancers. J Clin Oncol 2006, 24:2673-2678.

2. Wee DT, Thomas AA, Bradley PJ: Salivary duct carcinoma: what is already known, and can we improve survival? J Laryngol Otol 2012, 126(Suppl 2):S2-S7.

3. Kleinsasser O, Klein HJ, Hubner G: Salivary duct carcinoma. A group of salivary gland tumors analogous to mammary duct carcinoma. Arch Klin Exp Ohren Nasen Kehlkopfheilkd 1968, 192:100-105.

4. Adelstein DJ, Koyfman SA, El-Naggar AK, Hanna EY: Biology and management of salivary gland cancers. Semin Radiat Oncol 2012, 22:245-253.

5. McHugh JB, Visscher DW, Barnes EL: Update on selected salivary gland neoplasms. Arch Pathol Lab Med 2009, 133:1763-1774.

6. Lewis JE, McKinney BC, Weiland LH, Ferreiro JA, Olsen KD: Salivary duct carcinoma. Clinicopathologic and immunohistochemical review of 26 cases. Cancer 1996, 77:223-230.

7. Lagha A, Chraiet N, Ayadi M, Krimi S, Allani B, Rifi H, Raies H, Mezlini A Systemic therapy in the management of metastatic or advanced salivary gland cancers. Head Neck Oncol 2012, 4:19.

8. Jaehne M, Roeser K, Jaekel T, Schepers JD, Albert N, Loning T: Clinical and immunohistologic typing of salivary duct carcinoma: a report of 50 cases. Cancer 2005, 103:2526-2533.

9. Kaidar-Person O, Billan S, Kuten A: Targeted therapy with trastuzumab for advanced salivary ductal carcinoma: case report and literature review. Med Oncol 2012, 29:704-706.

10. Metzker ML: Sequencing technologies - the next generation. Nat Rev Genet 2010, 11:31-46.

11. Singh RR, Patel KP, Routbort MJ, Reddy NG, Barkoh BA, Handal B, KanagalShamanna R, Greaves WO, Medeiros LJ, Aldape KD, Luthra R: Clinical validation of a next-generation sequencing screen for mutational hotspots in 46 cancer-related genes. J Mol Diagn 2013, 15:607-622.

12. Karachaliou N, Mayo C, Costa C, Magri I, Gimenez-Capitan A, Molina-Vila MA, Rosell R: KRAS mutations in lung cancer. Clin Lung Canc 2013, 14:205-214.

13. Liu P, Cheng H, Roberts TM, Zhao JJ: Targeting the phosphoinositide 3-kinase pathway in cancer. Nat Rev Drug Discov 2009, 8:627-644. 
14. Courtney KD, Corcoran RB, Engelman JA: The PI3K pathway as drug target in human cancer. J Clin Oncol 2010, 28:1075-1083.

15. Gymnopoulos M, Elsliger MA, Vogt PK: Rare cancer-specific mutations in PIK3CA show gain of function. Proc Natl Acad Sci U S A 2007, 104:5569-5574.

16. Kato S, lida S, Higuchi T, Ishikawa T, Takagi Y, Yasuno M, Enomoto M, Uetake H, Sugihara K: PIK3CA mutation is predictive of poor survival in patients with colorectal cancer. Int J Canc 2007, 121:1771-1778.

17. Li SY, Rong M, Grieu F, lacopetta B: PIK3CA mutations in breast cancer are associated with poor outcome. Breast Cancer Res Treat 2006, 96:91-95.

18. Okudela K, Suzuki M, Kageyama S, Bunai T, Nagura K, Igarashi H, Takamochi K, Suzuki K, Yamada T, Niwa H, Ohashi R, Oqawa H, Mori H, Kitamura H, Kaneko T, Tsuneyoshi T, Suqimura H: PIK3CA mutation and amplification in human lung cancer. Pathol Int 2007, 57:664-671.

19. Nardi V, Sadow PM, Juric D, Zhao D, Cosper AK, Bergethon K, Scialabba VL, Batten JM, Borger DR, lafrate AJ, Heist RS, Lawrence DP, Flaherty KT, Bendell JC, Deschler D, Li Y, Wirth L, Dias-Santaqata D: Detection of novel actionable genetic changes in salivary duct carcinoma helps direct patient treatment. Clin Canc Res 2013, 19:480-490.

20. Qiu W, Tong GX, Turk AT, Close LG, Caruana SM, Su GH: Oncogenic PIK3CA mutation and dysregulation in human salivary duct carcinoma. BioMed Res Int 2014, 2014:810487.

21. Griffith CC, Seethala RR, Luvison A, Miller M, Chiosea SI: PIK3CA mutations and PTEN loss in salivary duct carcinomas. Am J Surg Pathol 2013, 37:1201-1207

22. Suda T, Hama T, Kondo S, Yuza Y, Yoshikawa M, Urashima M, Kato T, Moriyama $\mathrm{H}$ : Copy number amplification of the PIK3CA gene is associated with poor prognosis in non-lymph node metastatic head and neck squamous cell carcinoma. BMC Cancer 2012, 12:416.

23. Angulo B, Suarez-Gauthier A, Lopez-Rios F, Medina PP, Conde E, Tang M, Soler G, Lopez-Encuentra A, Cigudosa JC, Sanchez-Cespedes M: Expression signatures in lung cancer reveal a profile for EGFR-mutant tumours and identify selective PIK3CA overexpression by gene amplification. J Pathol 2008, 214:347-356.

24. Ettl T, Baader K, Stiegler C, Muller M, Agaimy A, Zenk J, Kuhnel T, Gosau M, Zeitler K, Schwarz S, Brockhoff G: Loss of PTEN is associated with elevated EGFR and HER2 expression and worse prognosis in salivary gland cancer. Br J Cancer 2012, 106:719-726.

25. Williams MD, Roberts DB, Kies MS, Mao L, Weber RS, El-Naggar AK: Genetic and expression analysis of HER-2 and EGFR genes in salivary duct carcinoma: empirical and therapeutic significance. Clin Canc Res 2010, 16:2266-2274.

26. Masubuchi T, Tada Y, Maruya SI, Osamura Y, Kamata SE, Miura K, Fushimi C, Takahashi H, Kawakita D, Kishimoto S, Nagao T: Clinicopathological significance of androgen receptor, HER2, Ki-67 and EGFR expressions in salivary duct carcinoma. Int J Clin Oncol 2014, [Epub ahead of print] PMID24553861.

27. Nabili V, Tan JW, Bhuta S, Sercarz JA, Head CS: Salivary duct carcinoma: a clinical and histologic review with implications for trastuzumab therapy. Head Neck 2007, 29:907-912.

28. Clauditz TS, Reiff M, Gravert L, Gnoss A, Tsourlakis MC, Munscher A, Sauter G, Bokemeyer C, Knecht R, Wilczak W: Human epidermal growth factor receptor 2 (HER2) in salivary gland carcinomas. Pathology 2011, 43:459-464.

29. Arteaga $C L$, Engelman JA: ERBB receptors: from oncogene discovery to basic science to mechanism-based cancer therapeutics. Cancer Cell 2014, 25:282-303.

30. Haddad R, Colevas AD, Krane JF, Cooper D, Glisson B, Amrein PC, Weeks L, Costello R, Posner M: Herceptin in patients with advanced or metastatic salivary gland carcinomas. A phase II study. Oral Oncol 2003, 39:724-727.

31. Slamon DJ, Leyland-Jones B, Shak S, Fuchs H, Paton V, Bajamonde A, Fleming T, Eiermann W, Wolter J, Pegram M, Baselqa J, Norton L: Use of chemotherapy plus a monoclonal antibody against HER2 for metastatic breast cancer that overexpresses HER2. N Engl J Med 2001, 344:783-792.

32. Glisson B, Colevas AD, Haddad R, Krane J, El-Naggar A, Kies M, Costello R, Summey C, Arquette M, Langer C, Amrein PC, Posner M: HER2 expression in salivary gland carcinomas: dependence on histological subtype. Clin Canc Res 2004, 10:944-946.

33. Johnson CJ, Barry MB, Vasef MA, Deyoung BR: Her-2/neu expression in salivary duct carcinoma: an immunohistochemical and chromogenic in situ hybridization study. Appl Immunohistochem Mol Morphol 2008, 16:54-58.

34. Falchook GS, Lippman SM, Bastida CC, Kurzrock R: Human epidermal receptor 2-amplified salivary duct carcinoma: regression with dual human epidermal receptor 2 inhibition and anti-vascular endothelial growth factor combination treatment. Head Neck 2014, 36:E25-E27.
35. Kumar B, Cordell KG, Lee JS, Worden FP, Prince ME, Tran HH, Wolf GT, Urba SG, Chepeha DB, Teknos TN, Eisbruch A, Tsien Cl, Taylor JM, D'Silva NJ, Yang K, Kurnit DM, Bauer JA, Bradford CR, Carey TE: EGFR, p16, HPV Titer, Bcl-xL and $\mathrm{p} 53$, sex, and smoking as indicators of response to therapy and survival in oropharyngeal cancer. J Clin Oncol 2008, 26:3128-3137.

36. Cohen EE, Rosen F, Stadler WM, Recant W, Stenson K, Huo D, Vokes EE: Phase II trial of ZD1839 in recurrent or metastatic squamous cell carcinoma of the head and neck. J Clin Oncol 2003, 21:1980-1987.

37. Locati LD, Bossi P, Perrone F, Potepan P, Crippa F, Mariani L, Casieri P, Orsenigo M, Losa M, Bergamini C, Liberatoscioli C, Quattrone P, Calderone RG Rinaldi G, Pilotti S, Licitra L: Cetuximab in recurrent and/or metastatic salivary gland carcinomas: A phase II study. Oral Oncol 2009, 45:574-578.

38. Hamakawa H, Nakashiro K, Sumida T, Shintani S, Myers JN, Takes RP, Rinaldo A, Ferlito A: Basic evidence of molecular targeted therapy for oral cancer and salivary gland cancer. Head Neck 2008, 30:800-809.

39. Jakob JA, Kies MS, Glisson BS, Kupferman ME, Liu DD, Lee JJ, El-Naggar AK, Gonzalez-Angulo AM, Blumenschein GR: A Phase II study of Gefitinib in patients with advanced salivary gland cancers. Head Neck 2014, [Epub ahead of print] PMID24585506.

40. Dahse R, Kosmehl H: Detection of drug-sensitizing EGFR exon 19 deletion mutations in salivary gland carcinoma. Br J Cancer 2008, 99:90-92.

\section{doi:10.1186/s12967-014-0299-6}

Cite this article as: Ku et al:: High-throughput profiling identifies

clinically actionable mutations in salivary duct carcinoma. Journal of Translational Medicine 2014 12:299.

\section{Submit your next manuscript to BioMed Central and take full advantage of:}

- Convenient online submission

- Thorough peer review

- No space constraints or color figure charges

- Immediate publication on acceptance

- Inclusion in PubMed, CAS, Scopus and Google Scholar

- Research which is freely available for redistribution

Submit your manuscript at www.biomedcentral.com/submit
C) Biomed Central 